\title{
Activity-Induced Radial Velocity Variation of M Dwarf Stars
}

\author{
Jan Marie Andersen ${ }^{1,2}$ and Heidi Korhonen ${ }^{2,3}$ \\ ${ }^{1}$ Department of Astronomy, Boston University, 725 Commonwealth Avenue, Boston, MA \\ 02215, USA, email: janmarie@bu.edu \\ ${ }^{2}$ Centre for Star and Planet Formation, Natural History Museum of Denmark, University of \\ Copenhagen, ster Voldgade 5-7, DK-1350, Copenhagen, Denmark \\ ${ }^{3}$ Niels Bohr Institute, University of Copenhagen, Juliane Maries Vej 30, DK-2100 Copenhagen, \\ Denmark
}

\begin{abstract}
Stellar magnetic activity manifests itself in a variety of ways including starspotscool, dark regions on the stellar surface. Starspots can cause variations ('jitter') in spectral line-profiles which can mimic the radial velocity (RV) variations caused by an orbiting planet, or create RV noise that can drown out a planetary signature. Cool, low-mass $\mathrm{M}$ dwarf stars can be highly active, which can make detection of potentially habitable planets around these stars difficult. We investigate radial velocity variations caused by different activity (spot) patterns on $\mathrm{M}$ dwarf stars in order to determine the limits of detectability for small planets orbiting active $\mathrm{M}$ dwarfs. We report on our progress toward the aim of answering the following questions: What types of spot patterns are realistic for $M$ dwarf stars? What effect will spots have on $M$ dwarf $R V$ measurements? Can jitter from $M$ dwarf spots mimic planetary signals? What is the ideal observing wavelength to reduce $M$ dwarf jitter?
\end{abstract}

Keywords. stars: activity, atmospheres, planetary systems, rotation, spots, low-mass; techniques: radial velocities

\section{Introduction}

In the search for habitable planets, M dwarfs have both been hailed as the Holy Grail of host stars and condemned as unsuitable targets. They are ubiquitous ( $\sim 70 \%$ of stars in the Milky Way are M dwarfs), and their low luminosities and temperatures combined with small masses should boost the radial velocity signatures of small planets in the 'habitable zone'. However, many $\mathrm{M}$ dwarfs are highly active, with long activity lifetimes (Hawley et al. 1996, West et al. 2008). This magnetic activity can be manifested in the form of dark, cool spots on the surface of the star. Such spots interfere with RV detection of exoplanets because they affect the measured RV signal from the star, creating false RV readings called 'jitter' which can mimic or drown out planetary signals (see Queloz et al. 2001). An example of this is CoRoT-7, a $\mathrm{K}$ dwarf that shows RV variations from both planets and stellar activity of the same order of magnitude. Different groups have concluded that the system contains one (Lèger et al. 2009), two (Queloz et al. 2009), or three planets (Hatzes et al. 2010). Pont et al. (2011) found weak confirmation of a first planet, CoRoT-7b, by analyzing the RV data, but concluded that the activity precludes the search for more planets beyond b. Ferraz-Mello et al. (2011) modeled the activity of the star and commented that "the activity of the star that contaminates the measured velocities not as a jitter, but as a dominant signal some two to three times more intense than the planetary contributions". Clearly, stellar activity is not to be ignored in RV analysis. 
The effects of starspots on RV measurements have also been studied by e.g. Makarov (2010), Boisse et al. (2010), and Boisse, Bonfils \& Santos (2012). Barnes, Jeffers, and Jones (2010) and Reiners et al. (2010) studied this effect focusing specifically on M dwarf stars. However, all of these studies are affected by a very limited knowledge of the behavior, amount, and patterns of spots on stellar surfaces, especially those of $\mathrm{M}$ dwarfs. Often for M dwarfs, solar models are extrapolated to "more active stars", and random/uniform spot coverage is assumed, which is not necessarily correct.

In this work we focus on the effects of starspots on $\mathrm{M}$ dwarf radial velocity measurements, with the goal of discovering the limits of detectability of potentially habitable planets orbiting $\mathrm{M}$ dwarf stars.

\section{Method}

We create spot patterns on a simulated stellar surface, from which spectral line profiles at different rotational phases of the star are calculated. Line profiles are generated using DIR7 (Piskunov, Tuominen \& Vilhu 1990; Hackman et al. 2001). For M dwarf photospheric temperatures we use the MARCS models (Gustafsson et al. 2008), a temperature grid of 2500 - $4000 \mathrm{~K}$, with a $100 \mathrm{~K}$ step size, and 20 limb angles. Radial velocity (jitter) measurements are obtained by cross-correlation of the calculated line profiles with a template profile, which is obtained from a spectrum with the temperature of the unspotted stellar surface. We developed code to generate spot (temperature) patterns on a stellar surface, with the capability of generating random spots across the entire surface, or in certain 'active' regions in latitude and longitude. Spots follow an approximately lognormal size distribution (Solanki et al. 1999), with an umbra temperature equal to that of the spot and penumbral temperature halfway between spot and photospheric temperatures. For M dwarfs we used a photospheric temperature of $3750 \mathrm{~K}$ and spot temperature of $2450 \mathrm{~K}$, although the temperatures and contrast ratios can be easily modified, which allows us to investigate stars of other spectral types.

Since the spot patterns on M dwarf stars are not well-known, we investigate cases with random, approximately uniform spot coverage, as well as stars that possess active regions-discrete areas on the stellar surface where spots are likely to form. Granzer et al. (2000) give the spot emergence latitude for ZAMS $0.4 \mathrm{M}_{\odot}$ star with different rotation rates. They find that on $\mathrm{M}$ dwarfs, spots are at least $20 \mathrm{deg}$ from the equator even at the slowest rotation rates. At higher rotation speeds, they see spots concentrated between $\sim 50$ to 70 degrees in latitude.

\section{Results and Discussion}

We investigated a cluster of spots in an active region at approximately 60 degrees latitude, with a $v \sin i=1.2 \mathrm{~km} / \mathrm{s}$ and inclination of $25 \mathrm{deg}$. The observed RV jitter resembles the RV curve generated by a large planet orbiting an M dwarf (see Fig. 1). We then used EXOFAST routines (Eastman et al. 2012) to fit these data to a planetary RV curve which yielded a stellar mass of $\mathrm{M}_{*}=0.33 \mathrm{M}_{\odot}$, a planetary mass of $\mathrm{M}_{P} /(\sin i)=$ $0.01 \mathrm{M}_{\text {Jupiter }}$ and a separation of $\mathrm{a}=0.01 \mathrm{AU}$.

To study the variation of RV jitter with wavelength, made RV calculations of a single, large spot with three different wavelength regions commonly used for M dwarf RV studies: $5000 \AA, 6400 \AA$ and $2.2 \mu m$ (see Fig. 2). The jitter decreases with increasing wavelength. 

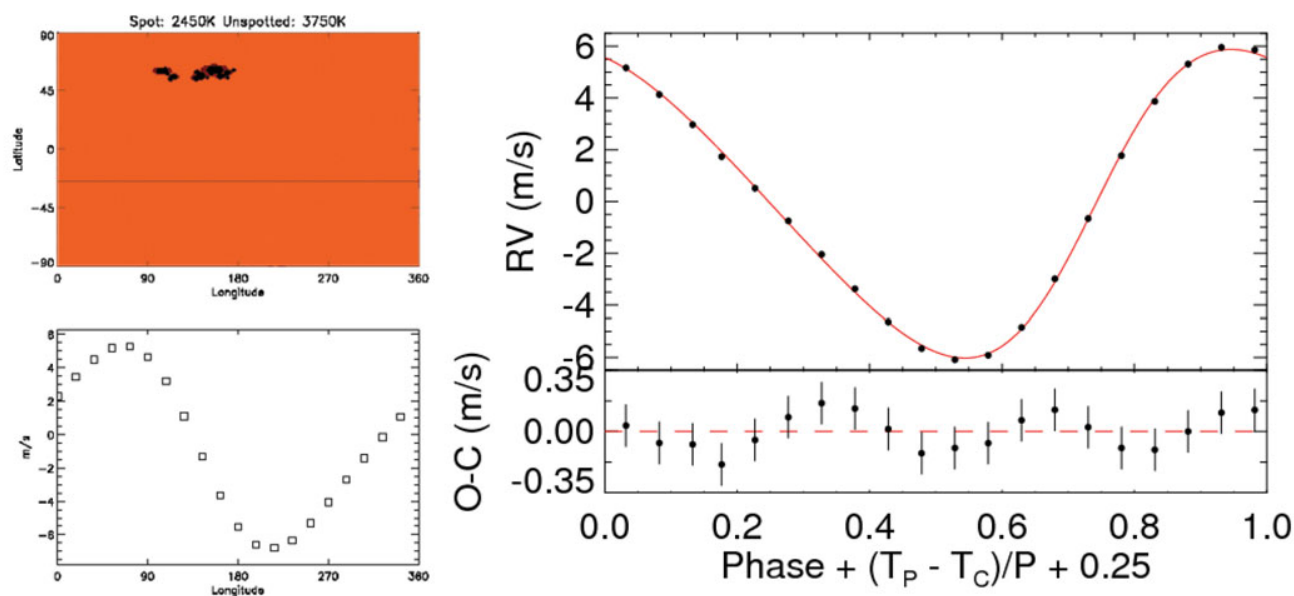

Figure 1. Spot cluster in an active region (top, left) can yield a radial velocity curve (bottom, left) similar to that of a planetary signature. Measured RV jitter from these spots was fit to a model planet using EXOFAST (Eastman et al. 2012), yielding a stellar mass of $\mathrm{M}=0.33 \mathrm{M}_{\odot}$ and a planetary mass of $\mathrm{M}_{P} /(\sin i)=0.01 \mathrm{M}_{\text {Jupiter }}$ in an orbit with a semi major axis of $\mathrm{a}=$ $0.01 \mathrm{AU}$ (right).
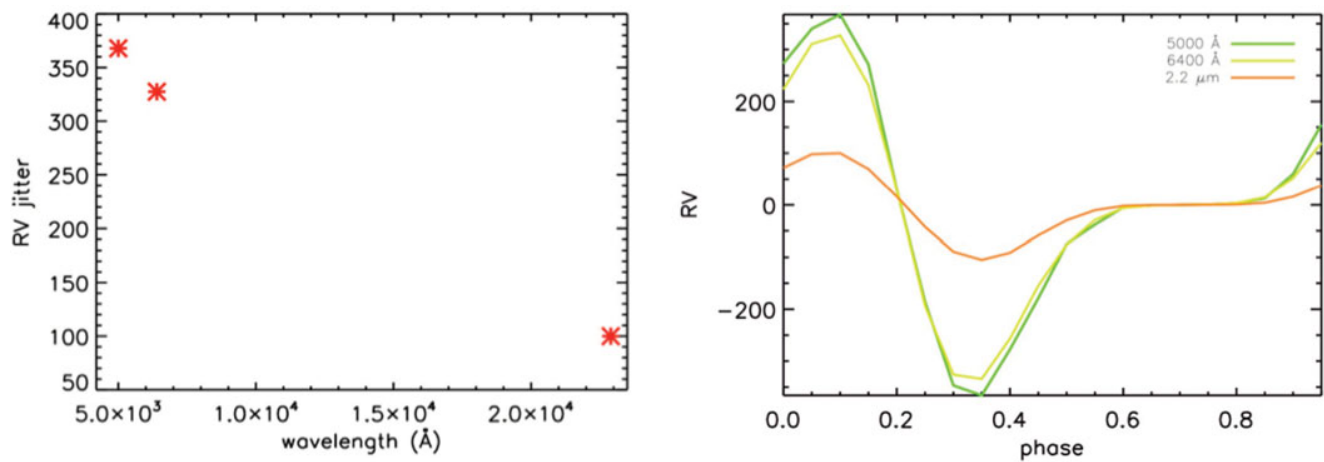

Figure 2. RV generated by a single, large spot at three different observational wavelengths (left). Semi-amplitude of RV jitter as a function of wavelength (right) shows that redder wavelengths reduce RV noise from spots.

\section{Conclusions}

This work improves upon previous studies by investigating both random/uniform spot configurations and stars with active latitude and longitude regions, and by attemptimg to create more realistic models of $\mathrm{M}$ dwarf spot configurations. One of the major obstacles in determining the RV noise caused by spots on $\mathrm{M}$ dwarfs is the lack of information on $\mathrm{M}$ dwarf spot configurations. The exact detection limits depend strongly both on the chosen spot configuration and the overall spottedness of the star. Thus we are attempting to create realistic spot maps based on models from the literature and the limited number of Doppler images of $\mathrm{M}$ dwarf spots, as well as simulating a large variety of different cases which will be presented in Andersen et al. 2013. 


\section{Acknowledgments}

J.M.A. acknowledges support through an NSF Graduate Research Fellowship and the Nordic Research Opportunity award, and travel grants from IAU and AAS. H.K. acknowledges the support from the European Commission under the Marie Curie IEF Programme in FP7, and an IAU travel grant to participate the General Assembly.

\section{References}

Barnes J. R., Jeffers S. V., \& Jones H. R. A. 2010, MNRAS, 412, 1599

Boisse, I., Bouchy, F., Hébrard, G., et al. 2011, A\&A, 528, A4

Boisse, I., Bonfils, X., \& Santos, N. C. 2012, A\&A, 545, A109

Eastman, J., Gaudi, B. S., \& Agol, E. 2012, PASP, 125, 83

Ferraz-Mello, S., Tadeu dos Santos, M., Beaug, C. et al. 2011, A\&SA, 531, A161

Granzer, T., Schüssler, M., Caligari, P., \& Strassmeier, K. G. 2000, A\&A, 355, 1087

Gustafsson, B., Edvardsson, B., Eriksson, K., et al. 2008, A\& 4 , 486, 951

Hackman, T., Jetsu, L., \& Tuominen, I. 2000, $A \& \& A, 374,171$

Hatzes A., Dvorak R., Wu chterl G., et al. 2010, A\& $A$, 520, A93

Lèger A., Rouan D., Schneider J, et al. 2009, A\& $A, 506,287$

Makarov, V. V. 2010, ApJ, 715, 500

Piskunov, N. E., Tuominen, I., \& Vilhu, O. 1990, A\&A, 230, 363

Pont, Frdric, Aigrain, Suzanne, \& Zucker, Shay 2011, MNRAS, 411, 1953

Queloz, D., Henry, G. W., Sivan, J. P., et al. 2001, A\&A, 379, 279

Queloz D., Bouchy F., Moutou C., et al. 2009, A\& $A$, 506, 303

Reiners, A., Bean, J. L., Huber, K. F., et al. 2010, ApJ, 710, 432

Solanki, S. K. 1999, Solar and Stellar Activity: Similarities and Differences, 158, 109

West, A. A., Hawley, S. L., Bochanski, J. J., et al. 2008, AJ, 135, 785 